\title{
Testing CPT Symmetry with Neutral K Mesons: A Review
}

\author{
Antonio Di Domenico \\ Dipartimento di Fisica, Sapienza Università di Roma \& INFN-Roma, P.le A. Moro, 5, I-00185 Rome, Italy; \\ antonio.didomenico@roma1.infn.it
}

Received: 3 November 2020; Accepted: 4 December 2020; Published: 11 December 2020

\begin{abstract}
The neutral kaon system is a very peculiar system that offers unique possibilities to perform precise tests of the CPT symmetry. The entanglement of neutral kaon pairs that are produced at a $\phi$-factory opens up new ways and scenarios in order to test this fundamental discrete symmetry. In this paper, the results of the most recent and significant CPT tests are reviewed. Experiments have set stringent limits on the CPT-violating parameters of different phenomenological models, some of them associated to possible decoherence mechanisms or Lorentz symmetry violation which might be justified in a quantum gravity framework. The present results show no violation of CPT symmetry, while their accuracy in some cases reaches the interesting level at which-in the most optimistic scenarios-quantum gravity effects might show up.
\end{abstract}

Keywords: CPT symmetry; discrete symmetries; neutral kaons; entanglement

\section{Introduction}

Rigorous proofs of the CPT theorem (with CPT the combination of charge conjugation (C), parity $(\mathrm{P})$, and time reversal $(\mathrm{T})$ transformations) were provided in the 50 's by the founding Fathers of the Quantum Field Theory [1-4] (also see Refs. [5-7] for some recent developments). The theorem ensures that exact CPT invariance holds for any quantum field theory that is formulated on flat space-time while assuming (1) Lorentz invariance, (2) Locality, and (3) Unitarity (i.e., conservation of probability). This theorem constitutes one of the most important results at the foundation of relativistic quantum field theory. It shares some of its foundational assumptions with another fundamental theorem: the Spin-Statistics Connection. Even though, on the surface, the two theorems appear to involve completely different subjects, they are, in fact, intimately linked, and both deeply connected to space-time symmetries $[8,9]$.

The latter constitutes an additional confirmation that testing the validity of CPT invariance means probing the most fundamental assumptions underpinning the Standard Model of elementary particles and their interactions. A violation of the CPT symmetry would have a dramatic impact and it would definitely constitute an unambiguous signal of a New Physics framework. Therefore, this constitutes a strong motivation for experimental and theoretical studies on this subject. In particular, in the last decades, in several attempts to discuss quantum gravity scenarios, speculative theoretical models have been considered that may exhibit a CPT-symmetry breakdown $[10,11]$.

Among the several systems in Nature, the neutral kaon doublet is one of the most intriguing, with its time evolution exhibiting rapid oscillations between particle and antiparticle states, and a variety of intricate interference phenomena. These, in turn, enable us to perform very refined tests of the fundamental principles of Quantum Mechanics, the interplay of different conservation laws, and the validity of various symmetry principles. In particular, the very high sensitivity of the neutral kaon system to several CPT-violating effects, which might arise in quantum gravity scenarios, makes it one of the best candidates for significant experimental tests of this symmetry [12]. For example, 
taking the precision on the fractional mass difference between the particle and anti-particle as a reference parameter for $\mathrm{CPT}$ violation effects, a precision of $\mathcal{O}\left(10^{-18}\right)$ can be reached for neutral kaons, while, for comparison, a limit of $\mathcal{O}\left(10^{-14}\right)$ can be reached for the $\mathrm{B}^{0}-\overline{\mathrm{B}}^{0}$ system, and only of $\mathcal{O}\left(10^{-10}\right)$ for proton-antiproton [13].

\section{The Neutral Kaon System}

The time evolution in the $\left\{\mathrm{K}^{0}, \overline{\mathrm{K}}^{0}\right\}$ space of a generic kaon state $|\mathrm{K}\rangle=a\left|\mathrm{~K}^{0}\right\rangle+b\left|\overline{\mathrm{K}}^{0}\right\rangle$ is governed by a Schrödinger-like equation with an effective Hamiltonian:

$$
\begin{aligned}
\mathbf{H} & =\left(\begin{array}{ll}
H_{11} & H_{12} \\
H_{21} & H_{22}
\end{array}\right)= \\
& =\mathbf{M}-\frac{i}{2} \boldsymbol{\Gamma}=\left(\begin{array}{ll}
m_{\mathrm{K}^{0}} & m_{12} \\
m_{12}^{*} & m_{\overline{\mathrm{K}}^{0}}
\end{array}\right)-\frac{i}{2}\left(\begin{array}{cc}
\Gamma_{\mathrm{K}^{0}} & \Gamma_{12} \\
\Gamma_{12}^{*} & \Gamma_{\overline{\mathrm{K}}^{0}}
\end{array}\right),
\end{aligned}
$$

where $\mathbf{M}$ and $\boldsymbol{\Gamma}$ are two hermitian matrices - the mass and decay matrices-and indices 1 and 2 stand for $\mathrm{K}^{0}$ and $\overline{\mathrm{K}}^{0}$ (with $m_{11}=m_{\mathrm{K}^{0}}$, etc.), respectively.

The eigenstates of $\mathbf{H}$ are the short- and long-lived physical states $\left|K_{S}\right\rangle$ and $\left|K_{L}\right\rangle$, which exhibit definite masses $m_{S, L}$ and lifetimes $\tau_{S, L}$ and they evolve as a function of the kaon proper time $t$, as:

$$
\begin{aligned}
\left|\mathrm{K}_{\mathrm{S}}(t)\right\rangle & =e^{-i \lambda_{S} t}\left|\mathrm{~K}_{\mathrm{S}}\right\rangle \\
\left|\mathrm{K}_{\mathrm{L}}(t)\right\rangle & =e^{-i \lambda_{L} t}\left|\mathrm{~K}_{\mathrm{L}}\right\rangle,
\end{aligned}
$$

with $\lambda_{S, L}=m_{S, L}-i \Gamma_{S, L} / 2$, and $\Gamma_{S, L}=\left(\tau_{S, L}\right)^{-1}$. They are usually expressed in terms of the flavor eigenstates $\left|\mathrm{K}^{0}\right\rangle,\left|\overline{\mathrm{K}}^{0}\right\rangle$, as:

$$
\begin{aligned}
& \left|\mathrm{K}_{\mathrm{S}}\right\rangle=\frac{1}{\sqrt{2\left(1+\left|\epsilon_{S}\right|^{2}\right)}}\left[\left(1+\epsilon_{S}\right)\left|\mathrm{K}^{0}\right\rangle+\left(1-\epsilon_{S}\right)\left|\overline{\mathrm{K}}^{0}\right\rangle\right] \\
& \left|\mathrm{K}_{\mathrm{L}}\right\rangle=\frac{1}{\sqrt{2\left(1+\left|\epsilon_{L}\right|^{2}\right)}}\left[\left(1+\epsilon_{L}\right)\left|\mathrm{K}^{0}\right\rangle-\left(1-\epsilon_{L}\right)\left|\overline{\mathrm{K}}^{0}\right\rangle\right],
\end{aligned}
$$

with $\epsilon_{S}$ and $\epsilon_{L}$ two small complex parameters describing CP impurity in the $\mathrm{K}^{0}-\overline{\mathrm{K}}^{0}$ mixing.

It is convenient to define the two parameters $\epsilon \equiv\left(\epsilon_{S}+\epsilon_{L}\right) / 2$, and $\delta \equiv\left(\epsilon_{S}-\epsilon_{L}\right) / 2$, which can be expressed in terms of the $\mathbf{H}$ matrix elements (ignoring the negligible quadratic terms) [12] :

$$
\begin{aligned}
& \epsilon=\frac{H_{12}-H_{21}}{2\left(\lambda_{S}-\lambda_{L}\right)}=\frac{-i \Im m_{12}-\frac{1}{2} \Im \Gamma_{12}}{\Delta m+i \Delta \Gamma / 2} \\
& \delta=\frac{H_{11}-H_{22}}{2\left(\lambda_{S}-\lambda_{L}\right)}=\frac{\frac{1}{2}\left(m_{\overline{\mathrm{K}}^{0}}-m_{\mathrm{K}^{0}}-\frac{i}{2}\left(\Gamma_{\overline{\mathrm{K}}^{0}}-\Gamma_{\mathrm{K}^{0}}\right)\right)}{\Delta m+i \Delta \Gamma / 2} .
\end{aligned}
$$

with $\Delta m=m_{L}-m_{S} \simeq 3.5 \times 10^{-15} \mathrm{GeV}$ and $\Delta \Gamma=\Gamma_{S}-\Gamma_{L} \simeq 7.0 \times 10^{-15} \mathrm{GeV}$. It is useful to define the so called superweak phase $\phi_{S W}=\arctan (2 \Delta m / \Delta \Gamma)$, and to adopt a suitable phase convention such that $\Im \Gamma_{12}=0$, fixing the phase of $\epsilon$ to $\phi_{S W}$, i.e., $\epsilon=|\epsilon| e^{i \phi_{S W}}$ (e.g., see Refs. [14,15]). Subsequently, it is easy to prove that:

- $\delta \neq 0$ implies CPT violation;

- $\epsilon \neq 0$ implies T violation; and,

- $\epsilon \neq 0$ or $\delta \neq 0$ implies CP violation.

It is worth noting here that the tiny value of the denominator of the r.h.s. expression in Equation (6) produces a strongly enhanced sensitivity of the parameter $\delta$ to possible CPT violation effects in the $\mathrm{K}^{0}-\overline{\mathrm{K}}^{0}$ mass and/or width difference at the numerator, similarly to the other oscillating neutral 
meson systems, $\mathrm{B}^{0}, \mathrm{~B}_{\mathrm{s}}^{0}$, and $\mathrm{D}^{0}$, where the enhancement in the analogous parameter is somewhat more moderate, due to the specific values of $\Delta m$ and $\Delta \Gamma$ for these systems.

\subsection{CPT Violation in Semileptonic Decays}

The semileptonic decay amplitudes have been conveniently parametrized in Refs. [16,17], as follows:

$$
\begin{aligned}
& \left\langle\pi^{-} l^{+} v|T| \mathrm{K}^{0}\right\rangle=a+b, \quad\left\langle\pi^{+} l^{-} \bar{\nu}|T| \overline{\mathrm{K}}^{0}\right\rangle=a^{*}-b^{*} \\
& \left\langle\pi^{+} l^{-} \bar{v}|T| \mathrm{K}^{0}\right\rangle=c+d, \quad\left\langle\pi^{-} l^{+} v|T| \overline{\mathrm{K}}^{0}\right\rangle=c^{*}-d^{*}
\end{aligned}
$$

with $a, b, c$, and $d$ complex quantities that are constrained by CP invariance ( $\Im a=\Re b=\Im c=\Re d=0$ ), $\mathrm{T}$ invariance $(\Im a=\Im b=\Im c=\Im d=0), \mathrm{CPT}$ invariance $(b=d=0)$, and $\Delta S=\Delta Q$ rule $(c=d=0)$. From their ratios, three observable parameters can be defined:

$$
y=-b / a, \quad x_{+}=c^{*} / a, \quad x_{-}=-d^{*} / a ;
$$

with y parametrizing CPT violation for $\Delta S=\Delta Q$ transitions, and $x_{+}\left(x_{-}\right)$describing the violation of the $\Delta S=\Delta Q$ rule in CPT invariant (violating) decay amplitudes. Subsequently, the observable semileptonic charge asymmetries for $K_{S}$ and $K_{L}$ states can be expressed as:

$$
\begin{aligned}
A_{S, L} & =\frac{\Gamma\left(\mathrm{K}_{\mathrm{S}, \mathrm{L}} \rightarrow \pi^{-} l^{+} v\right)-\Gamma\left(\mathrm{K}_{\mathrm{S}, \mathrm{L}} \rightarrow \pi^{+} l^{-} \bar{v}\right)}{\Gamma\left(\mathrm{K}_{\mathrm{S}, \mathrm{L}} \rightarrow \pi^{-} l^{+} v\right)+\Gamma\left(\mathrm{K}_{\mathrm{S}, \mathrm{L}} \rightarrow \pi^{+} l^{-} \bar{v}\right)} \\
& =2 \Re \epsilon \pm 2 \Re \delta-2 \Re y \pm 2 \Re x_{-} .
\end{aligned}
$$

Any difference between $A_{S}$ and $A_{L}$ signals a violation of the CPT symmetry:

$$
A_{S}-A_{L}=4\left(\Re \delta+\Re x_{-}\right) .
$$

\subsection{CPT Violation in Two Pion Decays}

The amplitudes of $\mathrm{K} \rightarrow \pi^{+} \pi^{-}, \pi^{0} \pi^{0}$ decays can be decomposed in terms of definite isospin $I=0,2$ of the final state:

$$
\begin{aligned}
\left\langle\pi \pi ; I|T| \mathrm{K}^{0}\right\rangle & =\left(A_{I}+B_{I}\right) e^{i \delta_{I}} \\
\left\langle\pi \pi ; I|T| \overline{\mathrm{K}}^{0}\right\rangle & =\left(A_{I}^{*}-B_{I}^{*}\right) e^{i \delta_{I}} .
\end{aligned}
$$

Here, $A_{I}\left(B_{I}\right)$ describe the CPT-invariant (CPT-violating) part of $\pi \pi$ decay amplitudes; $\delta_{I}$ is the $\pi \pi$ strong interaction phase shift for the channel of total isospin $I$. It is customary to define the ratio of decay amplitudes of $\mathrm{K}_{\mathrm{L}}$ and $\mathrm{K}_{\mathrm{S}}$ states, as:

$$
\begin{aligned}
\eta_{+-} & \equiv\left|\eta_{+-}\right| e^{i \phi_{+-}} \equiv \frac{\left\langle\pi^{+} \pi^{-}|T| \mathrm{K}_{\mathrm{L}}\right\rangle}{\left\langle\pi^{+} \pi^{-}|T| \mathrm{K}_{\mathrm{S}}\right\rangle}=\epsilon_{L}+i \frac{\Im A_{0}}{\Re A_{0}}+\frac{\Re B_{0}}{\Re A_{0}}+\epsilon^{\prime} \\
\eta_{00} & \equiv\left|\eta_{00}\right| e^{i \phi_{00}} \equiv \frac{\left\langle\pi^{0} \pi^{0}|T| \mathrm{K}_{\mathrm{L}}\right\rangle}{\left\langle\pi^{0} \pi^{0}|T| \mathrm{K}_{\mathrm{S}}\right\rangle}=\epsilon_{L}+i \frac{\Im A_{0}}{\Re A_{0}}+\frac{\Re B_{0}}{\Re A_{0}}-2 \epsilon^{\prime}
\end{aligned}
$$

where

$$
\epsilon^{\prime}=\frac{1}{\sqrt{2}} e^{i\left(\delta_{2}-\delta_{0}\right)} \frac{\Re A_{2}}{\Re A_{0}}\left[i\left(\frac{\Im A_{2}}{\Re A_{2}}-\frac{\Im A_{0}}{\Re A_{0}}\right)+\left(\frac{\Re B_{2}}{\Re A_{2}}-\frac{\Re B_{0}}{\Re A_{0}}\right)\right]
$$


$\epsilon^{\prime} \neq 0$ signals direct CP violation. It can be shown that (see Refs. [16-18]):

$$
\begin{aligned}
\phi_{00}-\phi_{+-} & \simeq \frac{3}{\sqrt{2}\left|\eta_{+-}\right|} \frac{\Re A_{2}}{\Re A_{0}}\left(\frac{\Re B_{2}}{\Re A_{2}}-\frac{\Re B_{0}}{\Re A_{0}}\right) \approx-3 \Im\left(\frac{\epsilon^{\prime}}{\epsilon}\right), \\
\phi_{+-}-\phi_{S W} & \simeq \frac{-1}{\sqrt{2}\left|\eta_{+-}\right|}\left(\frac{m_{\mathrm{K}^{0}}-m_{\overline{\mathrm{K}} 0}}{2 \Delta m}+\frac{\Re B_{0}}{\Re A_{0}}\right) .
\end{aligned}
$$

As a consequence, CPT violation in the $\pi \pi$ decay can manifest as a phase difference between the $\eta_{+-}$and $\eta_{00}$ parameters, while the difference between the phases $\phi_{+-}$and $\phi_{S W}$ can be due to a combination of CPT violation in the mixing and/or in the $\pi \pi$ decay.

The following quantity, which is obtained combining semileptonic and two pion decays parameters,

$$
\Re\left(\frac{2}{3} \eta_{+-}+\frac{1}{3} \eta_{00}\right)-\frac{A_{L}}{2}=\Re\left(y+x_{-}+\frac{\Re B_{0}}{\Re A_{0}}\right)
$$

signals CPT violation if different from zero.

The CPT-violating parameters that are presented in this Section, namely $\delta$ in the $\mathrm{K}^{0}-\overline{\mathrm{K}}^{0}$ mixing, and $y, x_{-}, B_{0}$, and $B_{2}$ in the decay amplitudes, have been considered to be constant quantities and do not entail any decoherence mechanism, Lorentz symmetry breaking, or other effects, which, instead, might be induced together with CPT violation in a quantum gravity phenomenology framework. Therefore, they are referred in the following as connected to standard CPT tests, to be distinguished from CPT violation effects in more elaborated scenarios that are briefly illustrated in the following.

\section{Experiments}

The most relevant results on CPT tests in the neutral kaon system mainly come from three experiments using different kaon beams, namely CPLEAR at CERN, KTeV at Fermilab, and KLOE at the INFN Frascati laboratory.

In the CPLEAR experiment [15], an antiproton beam is stopped in a gaseous hydrogen target. The subsequent antiproton annihilation process produces the following reactions with a branching fraction of about $0.4 \%$ :

$$
\begin{aligned}
& (\mathrm{p} \overline{\mathrm{p}}) \text { at rest } \\
& (\mathrm{p} \overline{\mathrm{p}}) \text { at rest }
\end{aligned}
$$

$\mathrm{K}^{-} \pi^{+}$or $\mathrm{K}^{+} \pi^{-}$are then selected to tag $\mathrm{K}^{0}$ or $\overline{\mathrm{K}}^{0}$ initial states, respectively.

The annihilation channel with a branching ratio of $0.07 \%$

$$
(\mathrm{p} \overline{\mathrm{p}})_{\text {at rest }} \rightarrow \mathrm{K}^{0}+\overline{\mathrm{K}}^{0}
$$

is instead used to study correlated $\mathrm{K}^{0} \overline{\mathrm{K}}^{0}$ pairs.

The whole CPLEAR detector is inside a solenoid providing a homogeneous longitudinal magnetic field of $0.44 \mathrm{~T}$. A series of cylindrical tracking detectors is devoted to the detection of charged particles, followed by a particle identification detector (PID) for charged kaon identification and electron/pion separation. The outermost detector is a six radiation lengths calorimeter that is used to detect photons from $\pi^{0}$ decays.

The CPLEAR detector collected a total of $1.1 \times 10^{13}$ antiproton interactions in the period from 1992 to 1996.

In the $\mathrm{KTeV}$ experiment [19] at Fermilab, an $800 \mathrm{GeV} / \mathrm{c}$ proton beam impinges on a beryllium oxide target, and two neutral kaon beams are formed from the secondary particles that are produced downstream the target while using a system of collimators, absorbers, and sweeping magnets. The neutral kaon decays are detected at a distance of about one hundred meters from the production 
target. One of the beams traverses an active regenerator, which is made of scintillator, producing a superposition of $\mathrm{K}_{\mathrm{S}}$ and $\mathrm{K}_{\mathrm{L}}$ states. The regenerator is alternatively swapped between the two beams in order to reduce the possible systematic effects.

A spectrometer instrumented with two drift chambers before and two after an analysing magnet measures the charged particle tracks and momenta, while neutral decay products are measured in a Cesium Iodine (CsI) crystal calorimeter that is located after the spectrometer. Background events for $\mathrm{K}_{\mathrm{S}, \mathrm{L}} \rightarrow \pi^{0} \pi^{0}$ coming from interactions in the regenerator, $\mathrm{K}_{\mathrm{L}} \rightarrow \pi^{0} \pi^{0} \pi^{0}$ or semileptonic decays are rejected by a hermetic veto system.

The KTeV results that are presented here refer to data that were collected in the periods 1996-1997 and 1999.

DA $\Phi N E$, the Frascati $\phi$-factory [20], is an $e^{+} e^{-}$collider running at the $\phi$ resonance peak, i.e., to a center of mass energy of $\sqrt{s} \sim 1020 \mathrm{MeV}$. The cross-section for the process $e^{+} e^{-} \rightarrow \phi$ is $\sim 3 \mu b$; the $\phi$-meson decay into $\mathrm{K}^{0} \overline{\mathrm{K}}^{0}$ has a branching fraction of $34 \%$. The neutral kaon pair is produced in an entangled quantum state with the same quantum numbers of the $\phi$-meson, $J^{P C}=1^{--}$:

$$
|i\rangle=\frac{1}{\sqrt{2}}\left\{\left|\mathrm{~K}^{0}\right\rangle\left|\overline{\mathrm{K}}^{0}\right\rangle-\left|\overline{\mathrm{K}}^{0}\right\rangle\left|\mathrm{K}^{0}\right\rangle\right\}=\frac{\mathcal{N}}{\sqrt{2}}\left\{\left|\mathrm{~K}_{\mathrm{S}}\right\rangle\left|\mathrm{K}_{\mathrm{L}}\right\rangle-\left|\mathrm{K}_{\mathrm{L}}\right\rangle\left|\mathrm{K}_{\mathrm{S}}\right\rangle\right\},
$$

where $\mathcal{N}=\sqrt{\left(1+\left|\epsilon_{S}\right|^{2}\right)\left(1+\left|\epsilon_{L}\right|^{2}\right)} /\left(1-\epsilon_{S} \epsilon_{L}\right) \simeq 1$ is a normalization factor.

Because of the entanglement in (18) and the huge difference in the lifetimes of the physical states $\left(\tau_{L} \gg \tau_{S}\right)$, the detection of a kaon at large times tags a $\left|K_{S}\right\rangle$ state in the opposite direction. This is a unique feature at a $\phi$-factory, which is not possible at other experimental facilities, related to a peculiar quantum effect, as discussed in Ref. [21], which has been exploited in the KLOE experiment in order to select pure $\mathrm{K}_{\mathrm{S}}$ beams.

The KLOE detector is constituted by a large volume drift chamber [22] that is surrounded by a scintillating fibre-lead electromagnetic calorimeter [23], both being immersed in an axial $0.52 \mathrm{~T}$ magnetic field that was provided by a superconducting coil. At KLOE, a $\mathrm{K}_{\mathrm{S}}$ state is tagged by identifying the distant interaction of the $\mathrm{K}_{\mathrm{L}}$ in the calorimeter $\left(\mathrm{K}_{\mathrm{L}}\right.$-crash) [21], while a $\mathrm{K}_{\mathrm{L}}$ decay in the drift chamber volume is tagged by detecting a $\mathrm{K}_{\mathrm{S}} \rightarrow \pi^{+} \pi^{-}$decay close to the interaction point (IP). KLOE completed the data taking in March 2006, with a total integrated luminosity of $\sim 2.5 \mathrm{fb}^{-1}$.

$D A \Phi N E$ has been subsequently upgraded implementing a crab-waist configuration $[24,25]$ for the colliding beams at IP, providing an effective improvement in the peak luminosity of approximately $60 \%$. The KLOE-2 experiment [26,27], which aimed to continue and extend the physics program of its predecessor, started data-taking in November 2014 at the upgraded DAФNE, with an improved KLOE detector. A total integrated luminosity of $\sim 5.5 \mathrm{fb}^{-1}$ has been collected by KLOE-2 after approximately three years of data-taking. In total, KLOE and KLOE-2 collected an integrated luminosity of $\sim 8 \mathrm{fb}^{-1}$, corresponding to $\sim 2.4 \times 10^{10} \phi$-mesons and $\sim 8 \times 10^{9} \mathrm{~K}_{\mathrm{S}} \mathrm{K}_{\mathrm{L}}$ entangled pairs produced. This constitutes a unique data sample, the largest existing worldwide at an $e^{+} e^{-}$collider running at the $\phi$-resonance peak, and it is very rich in Physics.

The KLOE-2 Physics program includes neutral kaon interferometry and tests of discrete symmetries and quantum mechanics, among the main issues. Because the KLOE results that are presented in the following Sections are dominated by the statistical uncertainties, the ongoing analyses exploiting the whole KLOE/KLOE-2 data sample are expected to significantly improve these results, as explained in detail in Refs. [12,26].

\section{4. "Standard" CPT Symmetry Tests}

In this section, the best experimental limits on the previously mentioned standard CPT-violating parameters are reviewed. 
The CPLEAR collaboration measured the following time-dependent decay rate asymmetry:

$$
A_{\delta}(\tau)=\frac{\bar{R}_{+}(\tau)-\alpha R_{-}(\tau)}{\bar{R}_{+}(\tau)+\alpha R_{-}(\tau)}+\frac{\bar{R}_{-}(\tau)-\alpha R_{+}(\tau)}{\bar{R}_{-}(\tau)+\alpha R_{+}(\tau)}
$$

where

$$
\begin{aligned}
& R_{+}(\tau)=R\left[\mathrm{~K}_{t=0}^{0} \rightarrow\left(\pi^{-} e^{+} v\right)_{t=\tau}\right], R_{-}(\tau)=R\left[\mathrm{~K}^{0}{ }_{t=0} \rightarrow\left(\pi^{+} e^{-} \bar{v}\right)_{t=\tau}\right] \\
& \bar{R}_{+}(\tau)=R\left[\overline{\mathrm{K}}_{t=0}^{0} \rightarrow\left(\pi^{-} e^{+} v\right)_{t=\tau}\right], \bar{R}_{-}(\tau)=R\left[\overline{\mathrm{K}}_{t=0}^{0} \rightarrow\left(\pi^{+} e^{-} \bar{v}\right)_{t=\tau}\right],
\end{aligned}
$$

and $\alpha=\left(1+4 \Re \epsilon_{L}\right)$. In the limit of large times, one has $A_{\delta}\left(\tau \gg \tau_{S}\right)=8 \Re \delta$, yielding the best measurement of $\Re \delta$ [28]:

$$
\Re \delta=\left(0.30 \pm 0.33_{\text {stat }} \pm 0.06_{\text {syst }}\right) \times 10^{-3} .
$$

The $\mathrm{KTeV}$ collaboration exploited the coherent regeneration phenomenon, occurring when a kaon beam traverses a slab of material, which modifies a pure $\mathrm{K}_{\mathrm{L}}$ beam into a coherent superposition of $\mathrm{K}_{\mathrm{L}}$ and $\mathrm{K}_{\mathrm{S}}$, i.e., $\left|\mathrm{K}_{\mathrm{L}}\right\rangle \rightarrow\left|\mathrm{K}_{\mathrm{L}}\right\rangle+\rho\left|\mathrm{K}_{\mathrm{S}}\right\rangle$, where $\rho$ is the regeneration (complex) parameter. The fit of the measured $\pi^{+} \pi^{-}$(and $\pi^{0} \pi^{0}$ ) decay intensity downstream the regenerator with the function

$$
\begin{aligned}
R_{+-(00)}(t) \propto & |\rho|^{2} e^{-\Gamma_{S} t}+\left|\eta_{+-(00)}\right|^{2} e^{-\Gamma_{L} t} \\
& +2|\rho|\left|\eta_{+-(00)}\right| e^{-\left(\Gamma_{S}+\Gamma_{L}\right) t / 2} \cos \left[\Delta m t+\phi(\rho)-\phi_{+-(00)}\right],
\end{aligned}
$$

yields the best CPT tests while using Equation (14) [19,29]:

$$
\begin{aligned}
\phi_{00}-\phi_{+-} & =(0.30 \pm 0.35)^{\circ} \\
\phi_{+-}-\phi_{S W} & =\left(0.61 \pm 0.62_{\text {stat }} \pm 1.01_{\text {syst }}\right)^{\circ} \\
\phi_{\epsilon}-\phi_{S W} & =(0.40 \pm 0.56)^{\circ}
\end{aligned}
$$

with $\phi_{\epsilon} \simeq\left(\phi_{00}+2 \phi_{+-}\right) / 3$, all being consistent with no CPT violation. $\mathrm{KTeV}$ also measured the asymmetry $A_{L}$ that is given in Equation (9) [30]

$$
A_{L}=\left(3322 \pm 58_{\text {stat }} \pm 47_{\text {syst }}\right) \times 10^{-6} .
$$

This result can be used in combination with two pion decay measurements in order to test CPT symmetry, as in Equation (15) [30]:

$$
\Re\left(y+x_{-}+\frac{\Re B_{0}}{\Re A_{0}}\right)=\Re\left(\frac{2}{3} \eta_{+-}+\frac{1}{3} \eta_{00}\right)-\frac{A_{L}}{2}=(-3 \pm 35) \times 10^{-6} .
$$

The measurement of the $\mathrm{K}_{\mathrm{S}}$ semileptonic charge asymmetry has been performed by the KLOE collaboration while analysing $\sim 2.1 \mathrm{fb}^{-1}$ of data [31]:

$$
A_{S}=\left(-3.8 \pm 5.0_{\text {stat }} \pm 2.6_{\text {syst }}\right) \times 10^{-3}
$$

Combining the results (24) and (26) for the charge asymmetries yields [31]:

$$
\begin{aligned}
& \left(A_{S}-A_{L}\right) / 4=(-1.8 \pm 1.4) \times 10^{-3} \\
& \left(A_{S}+A_{L}\right) / 4=(-0.1 \pm 1.4) \times 10^{-3} .
\end{aligned}
$$


Using the values of $\Re \delta$ and $\Re \epsilon$, the real part of the CPT violating parameters $y$ and $x_{-}$ (see Equation (8)) can be evaluated [31]:

$$
\begin{aligned}
\Re x_{-} & =\frac{A_{S}-A_{L}}{4}-\Re \delta=(-2.0 \pm 1.4) \times 10^{-3} \\
\Re y & =\Re \epsilon-\frac{A_{S}+A_{L}}{4}=(1.7 \pm 1.4) \times 10^{-3} .
\end{aligned}
$$

\section{CPT Test from Unitarity}

One of the most precise and significant standard CPT tests comes from the unitarity relation, which was originally derived by Bell and Steinberger [32]:

$$
\begin{aligned}
& \left(\frac{\Gamma_{S}+\Gamma_{L}}{\Gamma_{S}-\Gamma_{L}}+i \tan \phi_{S W}\right)\left[\frac{\Re \epsilon}{1+|\epsilon|^{2}}-i \Im \delta\right]= \\
& =\frac{1}{\Gamma_{S}-\Gamma_{L}} \sum_{f} \mathcal{A}^{*}\left(\mathrm{~K}_{\mathrm{S}} \rightarrow f\right) \mathcal{A}\left(\mathrm{K}_{\mathrm{L}} \rightarrow f\right) \equiv \sum_{f} \alpha_{f},
\end{aligned}
$$

where the sum runs over all accessible final states $f$ that appear in the decay amplitudes $\mathcal{A}\left(\mathrm{K}_{\mathrm{S}, \mathrm{L}} \rightarrow f\right)$. Equation (29) can be exploited to bound $\Im \delta$ (and other parameters), after having provided all of the $\alpha_{i}$ parameters, $\Gamma_{S}, \Gamma_{L}$, and $\phi_{S W}$ as inputs [13,33]:

$$
\Im \delta=(-0.3 \pm 1.4) \times 10^{-5}
$$

which is the most stringent limit on $\Im \delta$. The main limiting factors of this result are the uncertainties on $\pi \pi$ and semileptonic decay amplitudes.

The results on $\Im \delta$ and $\Re \delta$ are used in order to constrain the $\mathrm{K}^{0}-\overline{\mathrm{K}}^{0}$ mass and width differences. In the limit $\Gamma_{\mathrm{K}^{0}}-\Gamma_{\overline{\mathrm{K}}^{0}}=0$, i.e., neglecting the $C P T$-violating effects in the decay amplitudes, the stringent limit-as mentioned in the Introduction-on the neutral kaon mass difference is obtained [13]:

$$
\left|m_{\mathrm{K}^{0}}-m_{\overline{\mathrm{K}}^{0}}\right|<4.0 \times 10^{-19} \mathrm{GeV} \text { at } 95 \% \mathrm{CL} .
$$

It is interesting to compare the results for $\delta$ with the ones that were obtained for the analogous quantity $z=-2 \delta$ defined for the $\mathrm{B}^{0}$ system [13]:

$$
\Re z=(-4 \pm 4) \times 10^{-2}, \Im z=(-0.8 \pm 0.4) \times 10^{-2} .
$$

yielding a bound of $\mathcal{O}\left(10^{-14} \mathrm{GeV}\right)$ on the difference $\left|m_{\mathrm{B}^{0}}-m_{\overline{\mathrm{B}} 0}\right|[34]$.

\section{Tests of CPT Symmetry and Quantum Coherence}

At the DAФNE $\phi$-factory, the observable quantity is the double differential decay rate of the initial kaon pair state (18) into decay products $f_{1}$ and $f_{2}$ at proper times $t_{1}$ and $t_{2}$, respectively. The decay intensity after integration on $\left(t_{1}+t_{2}\right)$ at fixed time difference $\Delta t=t_{1}-t_{2}$, is given by [12]:

$$
\begin{gathered}
I\left(f_{1}, f_{2} ; \Delta t \geq 0\right)=C_{12}\left\{\left|\eta_{1}\right|^{2} e^{-\Gamma_{L} \Delta t}+\left|\eta_{2}\right|^{2} e^{-\Gamma_{S} \Delta t}\right. \\
\left.-2\left|\eta_{1}\right|\left|\eta_{2}\right| e^{-\frac{\left(\Gamma_{S}+\Gamma_{L}\right)}{2} \Delta t} \cos \left[\Delta m \Delta t+\phi_{2}-\phi_{1}\right]\right\}
\end{gathered}
$$

with

$$
\begin{aligned}
\eta_{i} & \equiv\left|\eta_{i}\right| e^{i \phi_{i}}=\frac{\left\langle f_{i}|T| \mathrm{K}_{\mathrm{L}}\right\rangle}{\left\langle f_{i}|T| \mathrm{K}_{\mathrm{S}}\right\rangle} \\
C_{12} & =\frac{|\mathcal{N}|^{2}}{2\left(\Gamma_{S}+\Gamma_{L}\right)}\left|\left\langle f_{1}|T| \mathrm{K}_{\mathrm{S}}\right\rangle\left\langle f_{2}|T| \mathrm{K}_{\mathrm{S}}\right\rangle\right|^{2} .
\end{aligned}
$$


This expression is valid for $\Delta t \geq 0$, while, for $\Delta t<0$, the substitutions $\Delta t \rightarrow|\Delta t|$ and $1 \leftrightarrow 2$ have to be applied.

The first evidence of the quantum interference in the time correlation of the two decays, as expressed in Equation (32) - peculiar of the initial entangled state (18) — has been observed by the KLOE collaboration in the CP violating channel $\phi \rightarrow \mathrm{K}_{\mathrm{S}} \mathrm{K}_{\mathrm{L}} \rightarrow \pi^{+} \pi^{-} \pi^{+} \pi^{-}$[35]. The measured $|\Delta t|$ distribution has been fitted with the function:

$$
I\left(\pi^{+} \pi^{-}, \pi^{+} \pi^{-} ;|\Delta t|\right) \propto e^{-\Gamma_{L}|\Delta t|}+e^{-\Gamma_{S}|\Delta t|}-2\left(1-\zeta_{S L}\right) e^{-\frac{\left(\Gamma_{S}+\Gamma_{L}\right)}{2}|\Delta t|} \cos (\Delta m|\Delta t|),
$$

where the quantum mechanical expression in the $\left\{\mathrm{K}_{\mathrm{S}}, \mathrm{K}_{\mathrm{L}}\right\}$ basis has been modified with the introduction of a decoherence parameter $\zeta_{S L}$ modulating the amplitude of the interference term, such that $\zeta_{S L}=0$ coincides with the quantum mechanical prediction and $\zeta_{S L}=1$ corresponds to total decoherence, i.e., to an incoherent mixture of $\mathrm{K}_{\mathrm{S}} \mathrm{K}_{\mathrm{L}}$ and $\mathrm{K}_{\mathrm{L}} \mathrm{K}_{\mathrm{S}}$ states. Analogously, a $\zeta_{0 \overline{0}}$ parameter is defined for the $\left\{\mathrm{K}^{0}, \overline{\mathrm{K}}^{0}\right\}$ basis [36]. The fit has been performed while taking the resolution and detection efficiency effects, the small contamination of non-resonant $e^{+} e^{-} \rightarrow \pi^{+} \pi^{-} \pi^{+} \pi^{-}$events, and the background due to coherent and incoherent $\mathrm{K}_{\mathrm{S}}$-regeneration on the beam pipe wall into account. The results of the analysis of a data sample that correspond to $L \sim 1.5 \mathrm{fb}^{-1}$ have been [35,37]:

$$
\begin{aligned}
& \zeta_{S L}=\left(0.3 \pm 1.8_{\text {stat }} \pm 0.6_{\text {syst }}\right) \times 10^{-2} \\
& \zeta_{0 \overline{0}}=\left(1.4 \pm 9.5_{\text {stat }} \pm 3.8_{\text {syst }}\right) \times 10^{-7},
\end{aligned}
$$

compatible with the prediction of quantum mechanics, i.e., $\zeta_{S L}=\zeta_{0 \overline{0}}=0$, and no decoherence effect. In particular, the result on $\zeta_{00}$ has a high precision, $\mathcal{O}\left(10^{-6}\right)$, due to the $\mathrm{CP}$ suppression that is present in this specific decay channel; it is an improvement by five orders of magnitude over the previous limit, obtained by Bertlmann and co-workers [36] in a re-analysis of CPLEAR data [38]. This result can also be compared to a similar one that was obtained in the $\mathrm{B}^{0}$ meson system with no $\mathrm{CP}$ suppression mechanism being present in the analysed channel, and an achieved precision of $\mathcal{O}\left(10^{-2}\right)$ for the decoherence parameter in the flavor basis: $\zeta_{\mathrm{B}^{0} \overline{\mathrm{B}}^{0}}=0.029 \pm 0.057$ [39].

The high precision of the $\zeta_{0 \overline{0}}$ result can be intuitively explained by the fact that the overall decay in which both kaons decay into $\pi^{+} \pi^{-}$, must be suppressed by $C P$ violation in the mixing, i.e., $|\epsilon|^{2} \sim 10^{-6}$, because one of the two kaons is a $\mathrm{K}_{\mathrm{L}}$. In quantum mechanics, this conclusion is independent on the basis that is used in order to express the initial state (18), while, in the case of a decoherence mechanism, it depends on the basis into which the initial state tends to spontaneously factorize. The decay $\mathrm{K}_{\mathrm{S}} \mathrm{K}_{\mathrm{L}} \rightarrow \pi^{+} \pi^{-}, \pi^{+} \pi^{-}$is still suppressed by $C P$ violation, while the decay $\mathrm{K}^{0} \overline{\mathrm{K}}^{0} \propto$ $\left(\mathrm{K}_{\mathrm{L}} \mathrm{K}_{\mathrm{S}}-\mathrm{K}_{\mathrm{S}} \mathrm{K}_{\mathrm{L}}+\mathrm{K}_{\mathrm{S}} \mathrm{K}_{\mathrm{S}}-\mathrm{K}_{\mathrm{L}} \mathrm{K}_{\mathrm{L}}\right) \rightarrow \pi^{+} \pi^{-}, \pi^{+} \pi^{-}$has a contribution from $\mathrm{K}_{\mathrm{S}} \mathrm{K}_{\mathrm{S}} \rightarrow \pi^{+} \pi^{-}, \pi^{+} \pi^{-}$that it is not suppressed, which explains the larger sensitivity to the decoherence effect in the case of the $\left\{\mathrm{K}^{0}, \overline{\mathrm{K}}^{0}\right\}$ basis, with respect to the $\left\{\mathrm{K}_{\mathrm{S}}, \mathrm{K}_{\mathrm{L}}\right\}$ basis.

Decoherence phenomena can be connected to models that are inspired by quantum gravity, in which space-time is subjected to inherent non-trivial topological quantum fluctuations at the Planck scale $\left(\sim 10^{-33} \mathrm{~cm}\right)$ with the the formation of microscopic event horizons. This structure of the space-time, generically called space-time foam, would cause pure states to evolve into mixed ones, i.e., to decoherence effects in apparently isolated physical systems [40]. A theorem proves that this kind of decoherence necessarily implies CPT violation, i.e., that the quantum mechanical operator generating CPT transformations is ill-defined [41].

Inspired by the above-mentioned decoherence mechanism, a specific model for decoherence that is based on the density matrix formalism and describing the time evolution of the neutral kaon system obeying a modified Liouville-von Neumann equation can be formulated [42,43]. In this model, three parameters, $\alpha, \beta$, and $\gamma$, signal the presence of decoherence and CPT violation effects. They are real, satisfy the inequalities $\alpha, \gamma>0$ and $\alpha \gamma>\beta^{2}$, and have mass units. 
The CPLEAR collaboration, studying single neutral kaon decays to $\pi^{+} \pi^{-}$and $\pi e v$ final states, obtained the results [44]:

$$
\begin{aligned}
& \alpha=(-0.5 \pm 2.8) \times 10^{-17} \mathrm{GeV} \\
& \beta=(2.5 \pm 2.3) \times 10^{-19} \mathrm{GeV} \\
& \gamma=(1.1 \pm 2.5) \times 10^{-21} \mathrm{GeV}
\end{aligned}
$$

The KLOE collaboration, which was studying the same $I\left(\pi^{+} \pi^{-}, \pi^{+} \pi^{-} ;|\Delta t|\right)$ distribution $\left(\mathrm{L} \sim 1.5 \mathrm{fb}^{-1}\right)$, as in the $\zeta$ parameters analysis in the simplifying hypothesis of complete positivity [45], i.e., $\alpha=\gamma$ and $\beta=0$, obtained the following result $[35,37]$ :

$$
\gamma=\left(0.7 \pm 1.2_{\text {stat }} \pm 0.3_{\text {syst }}\right) \times 10^{-21} \mathrm{GeV} .
$$

All of these results are compatible with no decoherence and CPT violation effects.

In the absence of a detailed microscopic theoretical model of quantum gravity, the exact dependence of the observable parameters $\alpha, \beta$, and $\gamma$ on the dimensionful constant $G_{N}=1 / M_{\text {Planck }}^{2}$, where $M_{\text {Planck }} \sim 1.2 \times 10^{19} \mathrm{GeV}$ is the Planck mass, and on the system-dependent quantities and numerical factors cannot be established.

Naively in a quantum gravity picture, one might expect CPT violation and the decoherence effects to be suppressed by a factor $E^{3} / M_{\text {Planck, }}^{2}$, with $E$ the typical energy scale of the low-energy probe. However, in some cases, e.g., in the context of non-critical string models for the space-time foam medium, resummation effects may result in much larger effects, of the order $E^{2} / M_{\text {Planck, }}$, as discussed in Refs. [46,47].

Given these considerations, what is worth underlining here is that, even without clear guidance from a detailed theoretical model, heuristically the CPT-violating and decoherence effects may appear-in the most optimistic scenarios for quantum gravity-at a scale of $\mathcal{O}\left(m_{K}^{2} / M_{\text {Planck }}\right) \sim 2 \times 10^{-20} \mathrm{GeV}$, which could be named as the interesting Planck scale region. The experimental sensitivity for the observable parameters, especially $\gamma$, turns out to be just in this range, and, therefore, can be considered of interest for testing ideas based on quantum gravity (the situation can be nicely depicted, as follows: a legendary treasure (an observable quantum gravity effect, e.g., the one related to the $\gamma$ parameter) from a sunken galleon is located deep into the sea, but at an unknown depth (the effective quantum gravity scale $M_{Q G}$ for this effect). To play this game of treasure hunt, you should start at least from the sea level (i.e., the most optimistic level for the hunt, corresponding to the interesting Planck scale region) and dive deep into the water. If you are lucky enough you may even find the treasure in shallow water. The treasure hunt above the sea level (i.e., in the air!) is not very exciting, you have no chance to find the treasure there)).

Quantitatively, one may go a small step further and assuming a certain power-law dependence easily translates the experimental results into corresponding limits on the effective quantum gravity scale at which these effects might show up. For instance, assuming $\gamma=\xi m_{K}^{2} / M_{\text {Planck }}$, from result (38), one gets $\xi<0.14$ at $90 \% \mathrm{CL}$ and a bound on the effective quantum gravity scale $M_{Q G}=M_{\text {Planck }} / \xi>8.7 \times 10^{19} \mathrm{GeV}$ at $90 \% \mathrm{CL}$, while, in the case of $\gamma \propto 1 / M_{\text {Planck }}^{2}$, a much looser bound is obviously obtained.

In a quantum gravity framework inducing decoherence, the CPT operator is ill-defined, as discussed above. This consideration has non-trivial consequences in entangled neutral kaon states. In fact, the resulting loss of particle-antiparticle identity might induce a modification of the correlation in state (18) that is imposed by Bose statistics [48,49]. Consequently, the initial state (18) is modified, as follows:

$$
|i\rangle=\frac{1}{\sqrt{2}}\left[\left|\mathrm{~K}^{0}\right\rangle\left|\overline{\mathrm{K}}^{0}\right\rangle-\left|\overline{\mathrm{K}}^{0}\right\rangle\left|\mathrm{K}^{0}\right\rangle+\omega\left(\left|\mathrm{K}^{0}\right\rangle\left|\overline{\mathrm{K}}^{0}\right\rangle+\left|\overline{\mathrm{K}}^{0}\right\rangle\left|\mathrm{K}^{0}\right\rangle\right)\right],
$$


where $\omega$ is a complex parameter describing a novel CPT violation phenomenon.

Similarly to the case that is discussed above for the $\alpha, \beta$ and $\gamma$ parameters, in the most optimistic scenario for quantum gravity, the order of magnitude of $|\omega|$ can be naively estimated to be [48,49]:

$$
|\omega| \sim\left[\left(m_{K}^{2} / M_{\text {Planck }}\right) / \Delta \Gamma\right]^{1 / 2} \sim 10^{-3} .
$$

In a more specific theoretical model of quantum gravity with stochastic space-time foam fluctuations, as described is Ref. [50], $|\omega|$, can be expressed as:

$$
|\omega|^{2} \sim \frac{\bar{\xi}^{2} k^{4}}{M_{\text {Planck }}^{2} \Delta m^{2}}
$$

with $k$ the kaon momentum and $\bar{\xi}$ a phenomenological parameter representing the momentum fraction transferred, on average, during the kaon interaction with the foam. In the absence of a complete theory of quantum gravity, $\bar{\zeta}$ cannot be further specified.

An analysis that is based on $\sim 1.5 \mathrm{fb}^{-1}$ of data performed by the KLOE collaboration on the CP violating $\phi \rightarrow \mathrm{K}_{\mathrm{S}} \mathrm{K}_{\mathrm{L}} \rightarrow \pi^{+} \pi^{-} \pi^{+} \pi^{-}$events, including in the fit of the $\Delta t$ distribution, the modified initial state Equation (39) yields the most precise measurement of the complex parameter $\omega[35,37]$ :

$$
\begin{aligned}
& \Re(\omega)=\left(-1.6_{-2.1 \text { stat }}^{+3.0} \pm 0.4_{\text {syst }}\right) \times 10^{-4} \\
& \Im(\omega)=\left(-1.7_{-3.0 \text { stat }}^{+3.3} \pm 1.2_{\text {syst }}\right) \times 10^{-4} .
\end{aligned}
$$

Here, we notice that, also in this case, the result is compatible with no CPT violation, while the experimental sensitivity is in the range of interest for testing quantum gravity in the most optimistic scenarios, and it could be used to loosely bound the phenomenological parameter $\bar{\xi}$ and the corresponding effective quantum gravity scale $M_{Q G}=M_{\text {Planck }} / \bar{\xi}$.

For comparison, in the case of entangled $\mathrm{B}^{0} \overline{\mathrm{B}}^{0}$ pairs, a reanalysis of data that were collected in the Babar experiment yields [51,52] $\Re\left(\omega_{\mathrm{B}^{0}}\right)=(0.8 \pm 4.6) \times 10^{-3}$.

\section{Test of CPT and Lorentz Symmetry}

CPT invariance holds for any realistic Lorentz-invariant quantum field theory. In recent years, attention has been drawn to the possibility that tiny violations of Lorentz symmetry could arise in a unified theory of gravity and quantum physics. While using effective field theory, a realistic and comprehensive description of Lorentz violation encompassing the non-abelian gauge symmetry of the Standard Model can be developed. The resulting framework is called the Standard-Model Extension (SME), providing a general description of CPT violation in a realistic field theory [53-56]. In the minimal SME scenario for neutral kaons, a CPT violation manifests to lowest order only in the mixing parameter $\delta$ (e.g., it vanishes at the first order in the decay amplitudes), and it exhibits a dependence on the four-momentum of the kaon [53-55]:

$$
\delta \approx i \sin \phi_{S W} e^{i \phi_{S W}} \gamma_{K}\left(\Delta a_{0}-\overrightarrow{\beta_{K}} \cdot \Delta \vec{a}\right) / \Delta m,
$$

where $\gamma_{K}$ and $\overrightarrow{\beta_{K}}$ are the kaon boost factor and velocity in the observer frame, and $\Delta a_{\mu}$ are four CPTand Lorentz-violating coefficients for the two valence quarks in the kaon. 
By studying the $\Delta t$ interference pattern of the entangled neutral kaon pairs in the decay channel $\phi \rightarrow \mathrm{K}_{\mathrm{S}} \mathrm{K}_{\mathrm{L}} \rightarrow \pi^{+} \pi^{-} \pi^{+} \pi^{-}$, as a function of sidereal time and particle direction in celestial coordinates, the KLOE collaboration obtained the following results while using $\sim 1.5 \mathrm{fb}^{-1}$ of data [57]:

$$
\begin{aligned}
\Delta a_{0} & =\left(-6.0 \pm 7.7_{\text {stat }} \pm 3.1_{\text {syst }}\right) \times 10^{-18} \mathrm{GeV} \\
\Delta a_{X} & =\left(0.9 \pm 1.5_{\text {stat }} \pm 0.6_{\text {syst }}\right) \times 10^{-18} \mathrm{GeV}, \\
\Delta a_{Y} & =\left(-2.0 \pm 1.5_{\text {stat }} \pm 0.5_{\text {syst }}\right) \times 10^{-18} \mathrm{GeV} \\
\Delta a_{Z} & =\left(3.1 \pm 1.7_{\text {stat }} \pm 0.5_{\text {syst }}\right) \times 10^{-18} \mathrm{GeV}
\end{aligned}
$$

These results constitute the most sensitive measurements in the quark sector of the SME. They can be compared to similar results that were obtained for the $B^{0}$ and $D^{0}$ mesons, where an accuracy of $\mathcal{O}\left(10^{-15} \mathrm{GeV}\right)$ and $\mathcal{O}\left(10^{-13} \mathrm{GeV}\right)$, respectively, has been reached [58,59].

\section{Direct CPT Tests in Transitions}

In Section 4 , the best limits on the $\delta$ parameter expressing CPT violation in the $\mathrm{K}^{0}-\overline{\mathrm{K}}^{0}$ mixing matrix have been reviewed, yielding a stringent limit on the difference $\left(m_{\mathrm{K}^{0}}-m_{\overline{\mathrm{K}}^{0}}\right)$. However, the tests of the CPT symmetry are often limited to the particle anti-particle difference of masses (or other intrinsic properties), i.e., to diagonal mass terms (like the survival probabilities presented in Equation (20)). In many physical phenomena, the violation effect does not appear at the first order in perturbation theory, being sufficient for the perturbation to break a symmetry of the non-perturbed states. This vanishing effect at first order for the diagonal elements, like, e.g., the case of the electric dipole moment for $\mathrm{T}$ violation, is not present for transitions (non-diagonal elements) [60].

Inspired by these considerations, a new kind of CPT symmetry tests that are based on (non-diagonal) transitions has been formulated for the neutral kaon system [61]. In this case, the necessary exchange of in and out states (and CP conjugation), is realised, exploiting the entanglement of the kaon pair that is produced at a $\phi$-factory. The methodology is similar to the one proposed for the tests of the T symmetry in the same context [62], or in the B meson system at B-factories $[51,60,63]$. The decay is not an essential ingredient for a non-vanishing effect and it is only used for filtering the appropriate initial and final states of the neutral kaon transition [64].

In order to implement this test, the entanglement of neutral kaons that are produced at a $\phi$-factory in the initial state (18) must be exploited. In fact, the perfect anticorrelation of the state in Equation (18), implies that it is possible to have a "flavor-tag" or a "CP-tag", i.e., to infer the flavor $\left(\mathrm{K}^{0}\right.$ or $\left.\overline{\mathrm{K}}^{0}\right)$ or the $\mathrm{CP}\left(\mathrm{K}_{+}\right.$or $\left.\mathrm{K}_{-}\right)$state of the still alive kaon by observing a specific flavor decay $\left(\pi^{+} \ell^{-} v\right.$ or $\left.\pi^{-} \ell^{+} \bar{v}\right)$ or $\mathrm{CP}$ decay $\left(\pi \pi\right.$ or $\left.\pi^{0} \pi^{0} \pi^{0}\right)$ of the other (and first decaying) kaon in the pair.

In this way, one can experimentally access-for instance-the transition $\mathrm{K}^{0} \rightarrow \mathrm{K}_{+}$, taken as reference, and the $\mathrm{K}_{+} \rightarrow \mathrm{K}^{0}, \overline{\mathrm{K}}^{0} \rightarrow \mathrm{K}_{+}$, and $\mathrm{K}_{+} \rightarrow \overline{\mathrm{K}}^{0}$ transitions, i.e., the $\mathrm{T}, \mathrm{CP}$, and CPT conjugated transitions, respectively. Specifically for the CPT symmetry test, one can directly compare the probabilities for the reference transition and the CPT conjugated one through the following ratios of probabilities:

$$
\begin{aligned}
& R_{1, C P T}(\Delta t)=P\left[\mathrm{~K}_{+}(0) \rightarrow \overline{\mathrm{K}}^{0}(\Delta t)\right] / P\left[\mathrm{~K}^{0}(0) \rightarrow \mathrm{K}_{+}(\Delta t)\right] \\
& R_{2, C P T}(\Delta t)=P\left[\mathrm{~K}^{0}(0) \rightarrow \mathrm{K}_{-}(\Delta t)\right] / P\left[\mathrm{~K}_{-}(0) \rightarrow \overline{\mathrm{K}}^{0}(\Delta t)\right] \\
& R_{3, C P T}(\Delta t)=P\left[\mathrm{~K}_{+}(0) \rightarrow \mathrm{K}^{0}(\Delta t)\right] / P\left[\overline{\mathrm{K}}^{0}(0) \rightarrow \mathrm{K}_{+}(\Delta t)\right] \\
& R_{4, C P T}(\Delta t)=P\left[\overline{\mathrm{K}}^{0}(0) \rightarrow \mathrm{K}_{-}(\Delta t)\right] / P\left[\mathrm{~K}_{-}(0) \rightarrow \mathrm{K}^{0}(\Delta t)\right] .
\end{aligned}
$$


CPT invariance imposes $R_{i, C P T}(\Delta t)=1$; any observed deviation from this prediction would signal CPT violation. It is worth noting that, for $\Delta t=0$ :

$$
R_{1, C P T}(0)=R_{2, C P T}(0)=R_{3, C P T}(0)=R_{4, C P T}(0)=1,
$$

i.e., the CPT-violating effect is built in the time evolution of the system, and it is absent at $\Delta t=0$.

For $\Delta t \gg \tau_{S}$, assuming the presence of CPT violation only in the mass matrix $(\delta \neq 0)$, one obtains:

$$
\begin{aligned}
& R_{2, C P T}\left(\Delta t \gg \tau_{S}\right) \simeq 1-4 \Re \delta \\
& R_{4, C P T}\left(\Delta t \gg \tau_{S}\right) \simeq 1+4 \Re \delta,
\end{aligned}
$$

i.e., the CPT-violating effect built in the time evolution reaches a "plateau" regime and dominates in this limit. It is a genuine effect, because $\Re \delta$ does not depend on $\Delta \Gamma$ as an essential ingredient [60,64].

At a $\phi$-factory, one can define two observable ratios:

$$
\begin{aligned}
R_{2, C P T}^{\exp }(\Delta t) & \equiv \frac{I\left(\ell^{-}, 3 \pi^{0} ; \Delta t\right)}{I\left(\pi \pi, \ell^{-} ; \Delta t\right)} \\
R_{4, C P T}^{\exp }(\Delta t) & \equiv \frac{I\left(\ell^{+}, 3 \pi^{0} ; \Delta t\right)}{I\left(\pi \pi, \ell^{+} ; \Delta t\right)},
\end{aligned}
$$

They are related to the $R_{i, C P T}(\Delta t)$ ratios that are defined in Equation (45), as follows, for $\Delta t \geq 0$ :

$$
\begin{aligned}
& R_{2, C P T}^{\exp }(\Delta t)=R_{2, C P T}(\Delta t) \times D_{C P T} \\
& R_{4, C P T}^{\exp }(\Delta t)=R_{4, C P T}(\Delta t) \times D_{C P T}
\end{aligned}
$$

whereas, for $\Delta t<0$, one has:

$$
\begin{aligned}
& R_{2, C P T}^{\exp }(\Delta t)=R_{1, C P T}(|\Delta t|) \times D_{C P T} \\
& R_{4, C P T}^{\exp }(\Delta t)=R_{3, C P T}(|\Delta t|) \times D_{C P T},
\end{aligned}
$$

with, to a high degree of accuracy, at least $\mathcal{O}\left(10^{-7}\right)$ :

$$
D_{C P T}=\frac{\left|\left\langle 3 \pi^{0}|T| \mathrm{K}_{-}\right\rangle\right|^{2}}{\left|\left\langle\pi \pi|T| \mathrm{K}_{+}\right\rangle\right|^{2}}=\frac{\mathrm{BR}\left(\mathrm{K}_{\mathrm{L}} \rightarrow 3 \pi^{0}\right)}{\mathrm{BR}\left(\mathrm{K}_{\mathrm{S}} \rightarrow \pi \pi\right)} \frac{\Gamma_{L}}{\Gamma_{S}} .
$$

One possible observable for the CPT test is constituted by the double ratio:

$$
\mathcal{D} \mathcal{R} \equiv \frac{R_{2, C P T}^{\exp }\left(\Delta t \gg \tau_{S}\right)}{R_{4, C P T}^{\exp }\left(\Delta t \gg \tau_{S}\right)},
$$

which is independent of $D_{C P T}$; it can be expressed as $\mathcal{D} \mathcal{R}=1-8 \Re \delta-8 \Re x_{-}$in the case of standard CPT violating effects.

Direct $C P$ violation in the decay amplitudes and violation of the $\Delta S=\Delta Q$ rule could, in principle, mimic CPT violation effects and weaken the significance of the test that is based on $\mathcal{D} \mathcal{R}$. However, it can be shown that they turn out to be totally negligible in the plateau region $\Delta t \gg \tau_{S}$, where $\mathcal{D} \mathcal{R}$ is defined (see detailed description in Ref. [61]). Therefore, the double ratio (54) constitutes one of the most robust and model independent observables for CPT tests, which could reveal possible new CPT violating mechanisms or further improve the precision of the present experimental limits.

KLOE and KLOE-2 data are being analysed to obtain the first measurement of the double ratio $\mathcal{D} \mathcal{R}$ with an expected statistical sensitivity of $\mathcal{O}\left(10^{-3}\right)$ [61]. 


\section{Conclusions}

Neutral kaons constitute a very special physical system for the study of discrete symmetries with unprecedented precision. The entanglement of neutral kaon pairs that are produced at a $\phi$-factory not only represents an ideal tool to push forward these studies, but also an excellent opportunity for testing the basic principles of Quantum Mechanics as well as the Lorentz symmetry.

The parameters that are related to several possible CPT violations effects, including decoherence and Lorentz-symmetry breaking effects, which might be justified in a quantum-gravity framework, have been measured. While the precision of the results in some cases reaches the interesting level at which quantum gravity effects might show up in the most optimistic scenarios, no violation of the CPT symmetry is observed.

The whole KLOE and KLOE-2 data sample is being analysed and improvements are expected in all of the KLOE results that are presented in this paper.

Finally, it is worth mentioning that, among the other oscillating neutral mesons, the $\mathrm{B}^{0}$ system seems to be the most suitable for testing CPT, despite presenting lower precision than with neutral kaons.

Funding: This research received no external funding.

Acknowledgments: The author would like to warmly thank the Scientific Editors for the invitation to contribute to this issue.

Conflicts of Interest: The author declares no conflict of interest.

\section{References}

1. Lüeders, G. Proof of the TCP Theorem. Ann. Phys. 1957, 2, 1-14; reprinted in Ann. Phys. 2000, 281, 1004-1018. [CrossRef]

2. Pauli, W. Exclusion principle, Lorentz group and reflexion of space-time and charge. In Niels Bohr and the Development of Physics; Pauli, W., Ed.; Pergamon: London, UK, 1955; pp. 30-51.

3. Bell, J.S. Time reversal in field theory. Proc. R. Soc. Lond. A 1955, 231, 479-495.

4. Jost, R. Eine Bemerkung zum CPT-theorem. Helv. Phys. Acta 1957, 30, 409-416.

5. Greenberg, O.W. CPT Violation Implies Violation of Lorentz Invariance. Phys. Rev. Lett. 2002, 89, 231602. [CrossRef] [PubMed]

6. Greenberg, O.W. Why is CPT Fundamental? Found. Phys. 2006,36, 1535-1553. [CrossRef]

7. Hollands, S. PCT Theorem for the Operator Product Expansion in Curved Spacetime. Commun. Math. Phys. 2004, 244, 209-244. [CrossRef]

8. Bain, J. CPT Invariance and the Spin-Statistics Connection; Oxford University Press: Oxford, UK, 2016.

9. Streater, R.F.; Wightman, A.S. PCT, Spin and Statistics, and All That; Princeton University Press: Princeton, NJ, USA, 1964.

10. Mavromatos, N.E. Decoherence and CPT Violation in a Stringy Model of Space-Time Foam. Found. Phys. 2010, 40, 917-960. [CrossRef]

11. Liberati, S. Tests of Lorentz invariance: A 2013 update. Class. Quantum Gravity 2013, 30, 133001. [CrossRef]

12. Di Domenico, A. Neutral kaon interferometry at a $\phi$-factory. In Handbook on Neutral Kaon Interferometry at a $\phi$-Factory; Di Domenico A., Ed.; Frascati Physics Series; INFN-LNF: Frascati, Italy, 2007; Volume 43, pp. 1-38.

13. Zyla, P.A.; Particle Data Group. Review of Particle Physics. Prog. Theor. Exp. Phys. 2020, 083C01. [CrossRef]

14. Fidecaro, M.; Gerber, H.J. The fundamental symmetries in the neutral kaon system-a pedagogical choice. Rep. Prog. Phys. 2006, 69 1713-1770. [CrossRef]

15. Angelopoulos, A. Physics at CPLEAR. Phys. Rep. 2003, 374, 165-270. [CrossRef]

16. Maiani, L. CP and CPT Violation in Neutral Kaon Decays. In The Second DAФNE Handbook; Maiani, L., Pancheri, G., Paver, N., Eds.; INFN-LNF: Frascati, Italy, 1995; Volume I, pp. 63-95.

17. D'Ambrosio, G.; Isidori G.; Pugliese, A. CP and CPT measurements at DAФNE. In The Second DAФNE Handbook; Maiani, L., Pancheri, G., Paver, N., Eds.; INFN-LNF: Frascati, Italy, 1995; Volume I, pp. 63-95. 
18. Buchanan, C.D.; Cousins, R.; Dib, C.; Peccei, R.D.; Quackenbush, J. Testing CP and CPT violation in the neutral kaon system at a $\phi$-factory. Phys. Rev. D 1992, 45 4088-4107. [CrossRef] [PubMed]

19. Alavi-Harati, A.; Alexopoulos, T.; Arenton, M.; Arisaka, K.; Averitte, S.; Barbosa, R.F.; Barker, A.R.; Barrio, M.; Bellantoni, L.; Bellavance, A.; et al. Measurements of direct CP violation, CPT symmetry, and other parameters in the neutral kaon system. Phys. Rev. D 2003 67, 012005. [CrossRef]

20. Gallo, A. DAФNE status report. Conf. Proc. 2006, C060626, 604-606.

21. Bernabeu, J.; Di Domenico, A. Can future observation of the living partner influence the past decayed state in entangled neutral K-mesons? arXiv 2019, arXiv:1912.04798v2.

22. Adinolfi, M.; Ambrosino, F.; Andryakov, A.; Antonelli, A. The tracking detector of the KLOE experiment. Nucl. Instrum. Methods A 2002, 488 51-73. [CrossRef]

23. Adinolfi, M.; Ambrosino, F.; Andryakov, A.; Antonelli, A. The KLOE electromagnetic calorimeter. Nucl. Instrum. Methods A 2002, 482, 364-386. [CrossRef]

24. Zobov, M. Test of crab-waist collisions at the DA $\Phi$ NE $\Phi$ factory. Phys. Rev. Lett. 2010, 104, 174801. [CrossRef]

25. Milardi, C.; Preger, M.A.; Raimondi, P.; Sgamma, F. High luminosity interaction region design for collisions inside high field detector solenoid. JINST 2012, 7, T03002. [CrossRef]

26. Amelino-Camelia, G. Physics with the KLOE-2 experiment at the upgraded DAФNE. Eur. Phys. J. C 2010, 68, 619-681. [CrossRef]

27. Di Domenico, A. KLOE-2 Workshop on $e^{+} e^{-}$Collision Physics at $1 \mathrm{GeV}$. Eur. Phys. J. Web Conf. $2018,166$. Availabe online: https:/ /www.epj-conferences.org/articles/epjconf/abs/2018/01/contents/contents.html (accessed on 4 December 2020).

28. Angelopoulos, A. A determination of the CPT violation parameter $\operatorname{Re}(\delta)$ from the semileptonic decay of strangeness-tagged neutral kaons. Phys. Lett. B 1998, 444, 52-60. [CrossRef]

29. Abouzaid, A.; Abouzaid, E.; Arenton, M.; Barker, A.R.; Barrio, M.; Bellantoni, L.; Blucher, E.; Bock, G.J. Precise measurements of direct CP violation, CPT symmetry, and other parameters in the neutral kaon system. Phys. Rev. D 2011, 83, 092001. [CrossRef]

30. Alavi-Harati, A. Measurement of the KL Charge Asymmetry. Phys. Rev. Lett. 2002, 88, 181601. [CrossRef]

31. Anastasi, A. Measurement of the charge asymmetry for the $\mathrm{K}_{\mathrm{S}} \rightarrow \pi e v$ decay and test of CPT symmetry with the KLOE detector. JHEP 2018, 09, 021. [CrossRef]

32. Bell, J.S.; Steinberger, J. Weak interactions of kaons. In Proceedings of the Oxford International Conference on Elementary Particles, Oxford, UK, 19-25 September 1965; Walsh, T.R., Taylor, A.E., Moorhouse, R.G., Southworth, B., Eds.; Rutherford High Energy Lab.: Chilton, UK, 1966; pp. 195-222.

33. Ambrosino, F. Determination of CP and CPT violation parameters in the neutral kaon system using the Bell-Steinberger relation and data from the KLOE experiment. JHEP 2006, 2006, 011.

34. Aubert, B. Limits on the decay-rate difference of neutral B mesons and on $\mathrm{CP}, \mathrm{T}$, and CPT violation in $\mathrm{B}^{0} \overline{\mathrm{B}}^{0}$ oscillations. Phys. Rev. D 2004, 70, 012007. [CrossRef]

35. Ambrosino, F. First observation of quantum interference in the process $\phi \rightarrow \mathrm{K}_{\mathrm{S}} \mathrm{K}_{\mathrm{L}} \rightarrow \pi^{+} \pi^{-} \pi^{+} \pi^{-}$: A Test of quantum mechanics and CPT symmetry. Phys. Lett. B 2006, 642, 315-321. [CrossRef]

36. Bertlmann, R.A.; Grimus, W.; Hiesmayr, B.C. Quantum mechanics, Furry's hypothesis, and a measure of decoherence in the $\mathrm{K}^{0} \overline{\mathrm{K}}^{0}$ system. Phys. Rev. D 1999, 60, 114032. [CrossRef]

37. Di Domenico, A. CPT symmetry and quantum mechanics tests in the neutral kaon system at KLOE. Found. Phys. 2010, 40, 852-866. [CrossRef]

38. Apostolakis A. An EPR experiment testing the nonseparability of the $K^{0}$ anti- $K^{0}$ wave function. Phys. Lett. $B$ 1998, 422, 339-348. [CrossRef]

39. Go, A.; Bay, A.; Abe, K. Measurement of Einstein-Podolsky-Rosen-Type Flavor Entanglement in $\mathrm{Y}(4 S) \rightarrow$ $B^{0} \overline{B^{0}}$ Decays. Phys. Rev. Lett. 2007, 99, 131802. [CrossRef] [PubMed]

40. Hawking, S. The Unpredictability of Quantum Gravity. Commun. Math. Phys. 1982, 87, 395-415. [CrossRef]

41. Wald, R. Quantum Gravity and Time reversibility. Phys. Rev. D 1980, 21, 2742-2755. [CrossRef]

42. Ellis, J.; Hagelin, J.S.; Nanopoulos, D.V.; Srednicki, M. Search for violations of quantum mechanics. Nucl. Phys. B 1984, 241, 381-405. [CrossRef]

43. Ellis, J.; Lopez, J.L.; Mavromatos, N.E.; Nanopoulos, D.V. Precision tests of CPT symmetry and quantum mechanics in the neutral kaon system. Phys. Rev. D 1996, 53, 3846-3870. [CrossRef]

44. Adler, R. Tests of CPT symmetry and quantum mechanics with experimental data from CPLEAR. Phys. Lett. B 1995, 364, 239-245. [CrossRef] 
45. Benatti, F.; Floreanini, F. Completely positive dynamical maps and the neutral kaon system. Nucl. Phys. B 1997, 488 335-363. [CrossRef]

46. Ellis, J.; Mavromatos, N.E.; Nanopoulos, D.V. String theory modifies quantum mechanics. Phys. Lett. B 1992, 292, 37-48. [CrossRef]

47. Ellis, J.; Mavromatos, N.E.; Nanopoulos, D.V. How large are dissipative effects in noncritical Liouville string theory? Phys. Rev. D 2000, 63, 024024. [CrossRef]

48. Bernabeu, J.; Mavromatos, N.; Papavassiliou, J. Novel Type of CPT Violation for Correlated Einstein-Podolsky-Rosen States of Neutral Mesons. Phys. Rev. Lett. 2004, 92, 131601. [CrossRef] [PubMed]

49. Bernabeu, J.; Mavromatos, N.E.; Papavassiliou, J.; Waldron-Lauda, A. Intrinsic CPT violation and decoherence for entangled neutral mesons. Nucl. Phys. B 2006, 744, 180-206. [CrossRef]

50. Bernabeu, J.; Mavromatos, N.E.; Sarkar, S. Decoherence induced CPT violation and entangled neutral mesons. Phys. Rev. D 2006, 74, 045014. [CrossRef]

51. Lees, J.P.; Poireau, V.; Tisserand, V.; Tico, J.G.; Grauges, E.; Palanoab, A.; Eigen, G.; Stugu, B.; Brown, D.N.; Kerth, L.T.; et al. Observation of Time-Reversal Violation in the $B^{0}$ Meson System. Phys. Rev. Lett. 2012, 109, 211801. [CrossRef]

52. Bernabeu, J.; Botella, F.J.; Mavromatos, N.E.; Nebot, M. The signal of ill-defined CPT weakening entanglement in the $B_{d}$ system. Eur. Phys. J. C 2017, 77, 865. [CrossRef] [PubMed]

53. Kostelecký, V.A. Sensitivity of CPT Tests with Neutral Mesons. Phys. Rev. Lett. 1998, 80, $1818-1821$. [CrossRef]

54. Kostelecký, V.A. Signals for CPT and Lorentz violation in neutral-meson oscillations. Phys. Rev. D 1999, 61, 016002. [CrossRef]

55. Kostelecký, V.A. CPT, T, and Lorentz violation in neutral-meson oscillations. Phys. Rev. D 2001, 64, 076001. [CrossRef]

56. Edwards, B.R.; Kostelecký, V.A. Searching for CPT violation with neutral-meson oscillations Phys. Lett. B 2019, 795, 620-626. [CrossRef]

57. Babusci, D.; Balwierz-Pytko, I.; Bencivenni, G.; Bloise, C.; Bossi, F.; Branchini, P.; Budano, A.; Balkestahl, L.C.; Capon, G.; Ceradini, F.; et al. Test of CPT and Lorentz symmetry in entangled neutral kaons with the KLOE experiment. Phys. Lett. B 2014, 730, 89-94. [CrossRef]

58. Kostelecký, V.A.; Russell, N. Data Tables for Lorentz and CPT Violation. Rev. Mod. Phys. 2011, 83, 11-31. 2020 edition, arXiv:0801.0287v13.

59. Aaij, R.; Beteta, C.A.; Adeva, B.; Adinolfi, M.; Ajaltouni, Z.; Akar, S.; Albrecht, J.; Alessio, F.; Alexander, M.; Ali, S.; et al. Search for violations of Lorentz invariance and CPT symmetry in $B_{(s)}^{0}$ mixing. Phys. Rev. Lett. 2016, 116, 241601. [CrossRef] [PubMed]

60. Bernabeu, J.; Martinez-Vidal, F. Colloquium: Time-reversal violation with quantum-entangled B mesons. Rev. Mod. Phys. 2015, 87, 165-182. [CrossRef]

61. Bernabeu, J.; Di Domenico, A.; Villanueva-Perez, P. Probing CPT in transitions with entangled neutral kaons. JHEP 2015, 10, 139. [CrossRef]

62. Bernabeu, J.; Di Domenico, A.; Villanueva-Perez, P. Direct test of time reversal symmetry in the entangled neutral kaon system at a $\phi$-factory. Nucl. Phys. B 2013, 868, 102-119. [CrossRef]

63. Bernabeu, J.; Martínez-Vidal, F.; Villanueva-Pérez, P. Time reversal violation from the entangled $B^{0} \bar{B}^{0}$ system. JHEP 2012, 08, 064. [CrossRef]

64. Wolfenstein, L. The search for direct evidence for time reversal violation. Int. J. Mod. Phys. E 1999, 8, 501-511. [CrossRef]

Publisher's Note: MDPI stays neutral with regard to jurisdictional claims in published maps and institutional affiliations. 